\title{
Mean First-Passage Time for Random Walks on Generalized Deterministic Recursive Trees
}

\author{
Francesc Comellas, Alicia Miralles \\ Dep. Matemàtica Aplicada IV, EPSC, \\ Universitat Politècnica de Catalunya \\ c/ Esteve Terradas 5, 08860 Castelldefels, Barcelona, Catalonia, Spain *
}

(Dated: January 11, 2010)

\begin{abstract}
We describe a technique that allows the exact analytical computation of the mean first passage time (MFPT) for infinite families of trees using their recursive properties. The method is based in the relationship between the MFPT and the eigenvalues of the Laplacian matrix of the trees but avoids their explicit computation. We apply this technique to find the MFPT for a family of generalized deterministic recursive trees. The method, however, can be adapted to other self-similar tree families.
\end{abstract}

PACS numbers: 89.20.Hh, 89.75.Fb, 89.75.Hc, 89.75.Da, 89.75.-k, 05.10.-a

\footnotetext{
${ }^{*}$ URL: http://www-ma4.upc.edu/ comellas; Electronic address: \{comellas, almirall\}@ma4.upc.es
} 


\section{INTRODUCTION}

In this past decade, the study of networks associated with complex systems has received the attention of researchers from many different areas. It has been shown that biological, social and economic networks, communication networks, the Internet and the www, as well as other artificial systems such as software architecture networks, are far from random and share interesting properties. In most cases, the networks have a low diameter (logarithmic with the number of nodes) and their degree distribution usually obeys either a power-law distribution (it is scale-free) or follows an exponential distribution [1]. Such properties can be often related to a modular or hierarchical structure and organization which is basic for their communication and dynamical processes. This hierarchical structure leads in many cases to the existence of nodes with a relatively high degree (or hubs), which play a critical role in the information flow of the system because many of the other nodes send and receive information through them $[2,3]$. Hubs are also associated with a low average distance in the network -the network is small-world-.

After an extensive initial research work centered mainly on network topological properties, a more recent approach using spectral techniques helps to understand dynamical processes associated with the networks. Among them, diffusion and in particular the characterization of random walks is a relevant topic. Related with random walks, mean first-passage time (MFPT) -the expected time that a random walker which starts with equal probability at any node will expend to reach a given target- is of interest, as it appears in important real-life first encounter events, which include network routing, reaction-diffusion processes, epidemic spreading, neuron firing, etc.; see [4-7].

Recent papers have considered the exact determination of the mean first-passage time for some self-similar network models, like the Sierpinski fractals [8, 9], pseudofractal web [10], Apollonian networks [11, 33], Koch networks [12], etc., including some trees as the iterative fractal scale-free network [13] or the T-graph [14, 15], The approach used considers the topology of the networks and employs decimation techniques or counting methods which usually require long and complex calculations. Here we provide a technique to compute the MFPT which is based on the relationship between the MFPT and the eigenvalues of the Laplacian matrix of the networks, but avoids their explicit computation. The method can be adapted to other recursive tree families. 


\section{LAPLACIAN SPECTRA OF A GRAPH AND MEAN FIRST PASSAGE TIME.}

For a given network, let $G=G(V, E)$ be its associated graph, with vertex set $V(n=|V|)$ and edge set $E(m=|E|)$. The Laplacian $\boldsymbol{L}$ of $G$ is a symmetric matrix with zero row-sums that accounts for the topology of the network, defined to be $L_{i j}=-1$ if nodes $i$ and $j$ are connected, $L_{i i}=\delta_{i}$ if node $i$ has degree $\delta_{i}$ (i.e. is connected to $\delta_{i}$ other nodes), and $L_{i j}=0$ otherwise. The Laplacian matrix is related to the adjacency matrix $\boldsymbol{A}$ of $G$ by $\boldsymbol{L}=\boldsymbol{D}-\boldsymbol{A}$, where $\boldsymbol{D}$ is the diagonal matrix of vertex degrees of $G$. The (Laplacian) spectrum of $G$ consists of the $n$ eigenvalues $\lambda_{1}, \lambda_{2}, \ldots, \lambda_{n}$ of the Laplacian matrix and they satisfy $0=\lambda_{1} \leq \lambda_{2} \leq \ldots, \leq \lambda_{n}[16]$.

The adjacency and Laplacian spectra of a graph are important as they provide bounds on its diameter, maximum and minimum degrees, and give information about possible partitions etc. They can also be used to count the number of paths of a given length in the network, number of triangles, total number of links, etc., see [16-18]. Dynamic properties of a network, like its synchronizability, could also be determined from the eigenvalues, see [19-21].

Except for particular families of graphs, usually with a high degree of symmetry (complete graphs, paths, stars, cycles, hypercubes, etc.), the determination of the exact spectrum of a general graph is a difficult task. However, for some large families of trees, the recursivity of the graph construction helps to find a relationship between the characteristic polynomials at different iteration steps [22-26], which can be used to produce iteratively an analytical expression for the spectra. Here, we apply a similar technique for the generalized deterministic recursive trees (GDRT) introduced in [24] and known as $r$-adic hypertrees. These trees generalize the deterministic uniform recursive tree (DURT) of Jung, Kim and Khang [27]. Although the exact determination of all the eigenvalues of GDRT is possible, we show that it is not needed to find the sum of the inverse of the eigenvalues greater than zero, which is used in the analytical determination of the mean first passage time of the network.

\section{First passage time and Kirchhoff index of a network}

For a connected graph $G(V, E)$, the first passage time (FPT) between vertices $i$ and $j$, denoted $F_{i j}$, is the time taken for a random walker on $G$ to reach vertex $j$ from vertex $i$. We consider here the mean first passage time $\langle F\rangle$ as the average of $F_{i j}$ over all pair of vertices.

The resistance distance $r_{i j}$ between two vertices $i$ and $j$ of a graph is defined as the effective (electrical) resistance between them when each edge of the graph has been replaced 
by a unit resistor. The Kirchhoff index $K f$ of a network, also called resistance, is defined as $K f=\sum_{i, j \in V ; i \neq j} r_{i j}$.

The FPT $F_{i j}$ between vertices has been related $[28,29]$ to the resistance distance as follows: $F_{i j}+F_{j i}=2 m r_{i j}$. For a tree, the Kirchhoff index is connected to the graph Laplacian eigenvalues as follows [30]

$$
K f=n \sum_{i=2}^{n} \frac{1}{\lambda_{i}}
$$

and as the mean first passage time for $G$ is, by definition,

$$
\langle F\rangle=\frac{1}{n(n-1)} \sum_{i, j \in V ; i \neq j} F_{i j}
$$

we can obtain the MFPT of a tree, where $m=n-1$, as

$$
\langle F\rangle=\sum_{i=2}^{n} \frac{1}{\lambda_{i}} .
$$

Therefore, for a tree, the mean first passage time can be obtained straightforwardly from the spectrum of its Laplacian. However, and as it has been stated, the analytical determination of this spectrum is difficult. Even the numerical calculation of the Laplacian eigenvalues is limited by the order (number of vertices) of the graph and non practical for large networks.

Next we show that, for trees which have a recursive structure, it is possible to compute the exact analytical MFPT as the sum of the inverse Laplacian eigenvalues (distinct from 0), without explicitly computing the eigenvalues. We illustrate the process with the generalized deterministic recursive trees introduced in [24].

\section{MEAN FIRST PASSAGE TIME FOR THE GENERALIZED DETERMINIS- TIC RECURSIVE TREE $T_{r, t}$}

The network considered in our study was introduced in [24] where its basic topological properties, including the degree distribution, were also found and the properties of the adjacency spectrum was studied. This tree was called $r$-adic hypertree of dimension $t$, and denoted as $T_{r, t}$. It is a generalization of the simplest instance of the deterministic uniform recursive tree introduced by Jung, Kim and Kahng in [27], and in this sense it can be called 

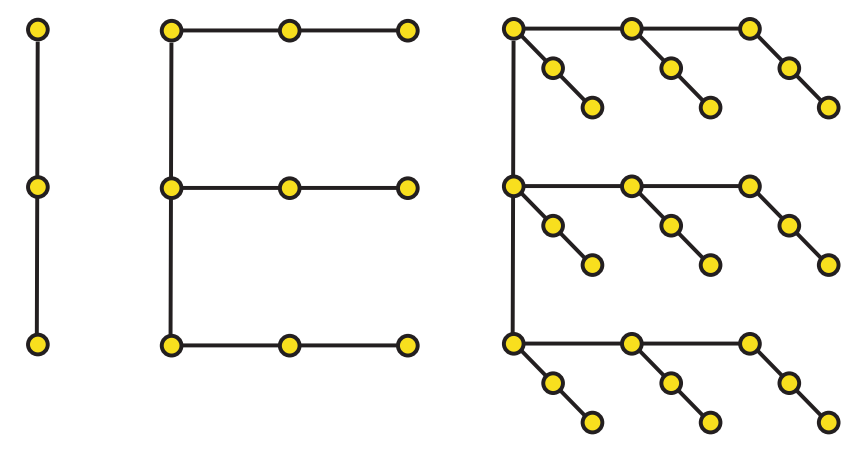

FIG. 1: The generalized deterministic recursive tree $T_{r, t}$ for $r=2$ and $t=0,1,2$.

generalized deterministic recursive tree, although this class of graphs contains other tree families.

It can be constructed in an iterative way as follows: For $t=0, T_{r, 0}$ is the path of $r$ nodes $P_{r}$. For $t \geq 1, T_{r, t}$ is obtained from $T_{r, t-1}$ by connecting a path $P_{r}$ to each node of $T_{r, t-1}$. Figure 1 shows $T_{r, 0}, T_{r, 1}$ and $T_{r, 2}$ for $r=3$. For $r=2$ we obtain the case $m=1$ of the deterministic uniform recursive tree given in [27]. The MFPT of the DURT has been studied by Zhang et al. in [26]. Here we study its generalization as GDRT and we give the exact analytical value for the MFPT for any value of $r$ and any iteration step $t$. A formal definition of $T_{r, t}$ as the $t$ hierarchical power of the path $P_{r}$ (with respect to the hierarchical product of graphs introduced in [23]), allowed its rigorous topological study in [24]. We adapt spectral techniques, likes those used for the study of the adjacency spectrum of the hierarchical product in [22-24], to analyze the Laplacian spectrum of the GDRT.

The eigenvalues of $T_{r, t}$

Let $\boldsymbol{A}_{t}$ and $\boldsymbol{D}_{t}$ denote the adjacency and diagonal degree matrices of $T_{r, t}$. (Note that to simplify the notation we avoid, in what follows, the use of the subscript $r$ wherever is possible.) We find that the adjacency matrix $\boldsymbol{A}_{t}$ and diagonal degree matrix $\boldsymbol{D}_{t}$ follow the 
relations [24]:

$$
\boldsymbol{A}_{t}=\left(\begin{array}{cccccc}
\boldsymbol{A}_{t-1} & \boldsymbol{I}_{t-1} & \mathbf{0} & \mathbf{0} & \cdots & \mathbf{0} \\
\boldsymbol{I}_{t-1} & \mathbf{0} & \boldsymbol{I}_{t-1} & \mathbf{0} & \cdots & \mathbf{0} \\
\mathbf{0} & \boldsymbol{I}_{t-1} & \mathbf{0} & \boldsymbol{I}_{t-1} & \cdots & \mathbf{0} \\
\mathbf{0} & \mathbf{0} & \boldsymbol{I}_{t-1} & \mathbf{0} & \cdots & \mathbf{0} \\
\vdots & \vdots & \vdots & \vdots & \vdots & \vdots \\
\mathbf{0} & \mathbf{0} & \cdots & \mathbf{0} & \boldsymbol{I}_{t-1} & \mathbf{0}
\end{array}\right) \text { and } \boldsymbol{D}_{t}=\left(\begin{array}{cccccc}
\boldsymbol{D}_{t-1}+\boldsymbol{I}_{t-1} & \mathbf{0} & \mathbf{0} & \cdots & \mathbf{0} & \mathbf{0} \\
\mathbf{0} & 2 \boldsymbol{I}_{t-1} & \mathbf{0} & \cdots & \mathbf{0} & \mathbf{0} \\
\mathbf{0} & \mathbf{0} & 2 \boldsymbol{I}_{t-1} & \cdots & \mathbf{0} & \mathbf{0} \\
\vdots & \vdots & \vdots & \vdots & \vdots & \vdots \\
\mathbf{0} & \mathbf{0} & \mathbf{0} & \cdots & 2 \boldsymbol{I}_{t-1} & \mathbf{0} \\
\mathbf{0} & \mathbf{0} & \mathbf{0} & \cdots & \mathbf{0} & \boldsymbol{I}_{t-1}
\end{array}\right)
$$

and both are $r \times r$ block matrices with $\boldsymbol{A}_{t-1}, \boldsymbol{D}_{t-1}$ being $r^{t} \times r^{t}$ matrices associated with the tree $T_{r, t-1}$ and $\boldsymbol{I}_{t-1}$ is the identity matrix with the same dimensions.

Thus, the following recursive relation between the Laplacian matrices $\boldsymbol{L}_{t}$ and $\boldsymbol{L}_{t-1}$ holds:

$$
\boldsymbol{L}_{t}=\boldsymbol{D}_{t}-\boldsymbol{A}_{t}=\left(\begin{array}{cccccc}
\boldsymbol{L}_{t-1} & -\boldsymbol{I}_{t-1} & \mathbf{0} & \cdots & \mathbf{0} & \mathbf{0} \\
-\boldsymbol{I}_{t-1} & 2 \boldsymbol{I}_{t-1} & -\boldsymbol{I}_{t-1} & \cdots & \mathbf{0} & \mathbf{0} \\
\mathbf{0} & -\boldsymbol{I}_{t-1} & 2 \boldsymbol{I}_{t-1} & \cdots & \mathbf{0} & \mathbf{0} \\
\vdots & \vdots & \vdots & \vdots & \vdots & \vdots \\
\mathbf{0} & \mathbf{0} & \mathbf{0} & \cdots & 2 \boldsymbol{I}_{t-1} & -\boldsymbol{I}_{t-1} \\
\mathbf{0} & \mathbf{0} & \mathbf{0} & \cdots & -\boldsymbol{I}_{t-1} & \boldsymbol{I}_{t-1}
\end{array}\right)
$$

Next we provide a recursive relationship which allows to find the Laplacian eigenvalues of $T_{r, t}$ from those of $T_{r, t-1}$. To obtain the spectrum of $T_{r, t}$, we need to solve the equation $\boldsymbol{L}_{t} \boldsymbol{x}=\lambda \boldsymbol{x}$. We can write this equation as follows:

$$
\begin{array}{cccccc}
\left(\boldsymbol{L}_{t-1}+\boldsymbol{I}_{t-1}\right) \boldsymbol{x}_{1}-\boldsymbol{I} \boldsymbol{x}_{2} & & & & \\
-\boldsymbol{I}_{t-1} \boldsymbol{x}_{1} & +2 \boldsymbol{I}_{t-1} \boldsymbol{x}_{2} & -\boldsymbol{I}_{t-1} \boldsymbol{x}_{3} & & \lambda \boldsymbol{x}_{1} \\
& -\boldsymbol{I}_{t-1} \boldsymbol{x}_{2} & +2 \boldsymbol{I}_{t-1} \boldsymbol{x}_{3} & -\boldsymbol{I}_{t-1} \boldsymbol{x}_{4} & = & \lambda \boldsymbol{x}_{3} \\
\vdots & & \vdots & \vdots & \vdots \\
& & \vdots & \vdots & \\
& -\boldsymbol{I}_{t-1} \boldsymbol{x}_{r-2} & +2 \boldsymbol{I}_{t-1} \boldsymbol{x}_{r-1} & -\boldsymbol{I}_{t-1} \boldsymbol{x}_{r} & = & \lambda \boldsymbol{x}_{r-1} \\
& & -\boldsymbol{I}_{t-1} \boldsymbol{x}_{r-1} & +\boldsymbol{I}_{t-1} \boldsymbol{x}_{r} & = & \lambda \boldsymbol{x}_{r}
\end{array}
$$

where $\boldsymbol{x}=\left(\boldsymbol{x}_{1}, \boldsymbol{x}_{2}, \cdots, \boldsymbol{x}_{r}\right)^{T}$ and each $\boldsymbol{x}_{i}, 1 \leq i \leq r$, has $r^{t}$ elements.

If $\lambda^{*}$ is an eigenvalue of $\boldsymbol{L}_{t-1}, \boldsymbol{L}_{t-1} \boldsymbol{x}_{1}=\lambda^{*} \boldsymbol{x}_{1}$ holds. Therefore we may write the system 
of Eq. (2) as:

$$
\begin{aligned}
\left(\lambda^{*}+1\right) \boldsymbol{x}_{1}-\boldsymbol{x}_{2} & =\lambda \boldsymbol{x}_{1} \\
-\boldsymbol{x}_{1}+2 \boldsymbol{x}_{2}-\boldsymbol{x}_{3}= & \lambda \boldsymbol{x}_{2} \\
-\boldsymbol{x}_{2}+2 \boldsymbol{x}_{3}-\boldsymbol{x}_{4}= & \lambda \boldsymbol{x}_{3} \\
\vdots & \vdots \\
-\boldsymbol{x}_{r-2}+2 \boldsymbol{x}_{r-1}-\boldsymbol{x}_{r}= & \lambda \boldsymbol{x}_{r-1} \\
-\boldsymbol{x}_{r-1}+\boldsymbol{x}_{r} & =\lambda \boldsymbol{x}_{r}
\end{aligned}
$$

We solve this system by substituting back from the last equation and we obtain

$$
\left(\lambda-1-\frac{1}{(\lambda-2)-\frac{1}{(\lambda-2)-\frac{1}{\vdots_{(\lambda-2)-\frac{1}{(\lambda-1)}}}}}-\lambda^{*}\right) \boldsymbol{x}_{1}=0
$$

If we define

$$
R_{r}(\lambda)=(\lambda-1)-\frac{1}{(\lambda-2)-\frac{1}{(\lambda-2)-\frac{1}{\vdots_{(\lambda-2)-\frac{1}{(\lambda-1)}}}}}
$$

we have

$$
R_{r}(\lambda)=\lambda_{i}^{t-1}, \quad 1 \leq i \leq r^{t}
$$

This equation, for each of the $r^{t}$ eigenvalues of $\boldsymbol{L}_{t-1}$, gives $r$ distinct eigenvalues of $\boldsymbol{L}_{t}$.

The recursion for the spectrum given by Eq. (4) allows the iterative calculation of the full Laplacian spectrum of $T_{r, t}$. Note that this spectrum consists of $r^{t+1}$ distinct eigenvalues which we can write $s p\left(T_{r, t}\right)=\left\{\lambda_{1}^{t}, \lambda_{2}^{t}, \ldots, \lambda_{r+1}^{t}\right\}$.

We recall now that the mean first passage time, $\langle F\rangle_{t}$, for the tree $T_{r, t}$ can be obtained as the sum of the inverse of all its Laplacian eigenvalues (except 0). We label the eigenvalues of $T_{r, 0}=P_{r}$ as $\lambda_{i}^{0}, 1 \leq i \leq r$, and for convenience let us consider that $\lambda_{0}^{0}$ is the eigenvalue 0 . For each eigenvalue at step $t-1, \lambda_{i}^{t-1}, 1 \leq i \leq r^{t}$, Eq. (4) generates, at step $t, r$ distinct eigenvalues, which we denote $\lambda_{i, 1}^{t}, \lambda_{i, 2}^{t}, \cdots, \lambda_{i, r}^{t}$. Therefore, for $r \geq 2$ and $t \geq 1$, we can write the mean first passage time of $T_{r, t}$ as:

$$
\langle F\rangle_{t}=\sum_{i=1}^{r^{t}-1}\left(\frac{1}{\lambda_{i, 1}^{t}}+\frac{1}{\lambda_{i, 2}^{t}}+\cdots+\frac{1}{\lambda_{i, r}^{t}}\right)+\langle F\rangle_{0},
$$

where $\langle F\rangle_{0}=\sum_{i=2}^{r} \frac{1}{\lambda_{i}^{0}}$. 
To calculate this expression we analyze $R(\lambda)$. From its definition in Eq. (3) and for $r>2$, we have

$$
R_{r}(\lambda)=\lambda-1-\frac{1}{R_{r-1}(\lambda)-1}
$$

and introducing polynomials $N_{r}(\lambda)$ and $D_{r}(\lambda)$ such that $R_{r}(\lambda)=\frac{N_{r}(\lambda)}{D_{r}(\lambda)}$ we can write

$$
R_{r}(\lambda)=\frac{N_{r}(\lambda)}{D_{r}(\lambda)}=\lambda-1-\frac{1}{\frac{N_{r-1}(\lambda)}{D_{r-1}(\lambda)}-1}=\frac{\left(N_{r-1}(\lambda)-D_{r-1}(\lambda)\right) \lambda-N_{r-1}(\lambda)}{N_{r-1}(\lambda)-D_{r-1}(\lambda)}
$$

which leads, using Eq. (4), to

$$
N_{r}(\lambda)-\lambda_{i}^{t-1} D_{r}(\lambda)=0
$$

We consider the following recurrences and initial conditions:

$$
\begin{aligned}
& N_{r}(\lambda)=\left(N_{r-1}(\lambda)-D_{r-1}(\lambda)\right) \lambda-N_{r-1}(\lambda), N_{2}(\lambda)=\lambda(\lambda-2) \\
& D_{r}(\lambda)=N_{r-1}(\lambda)-D_{r-1}(\lambda), D_{2}(\lambda)=\lambda-1
\end{aligned}
$$

and for $r \geq 2$, after some elementary calculations, we obtain that:

$$
N_{r}(0)=0, \quad D_{r}(0)=(-1)^{r-1}
$$

and, if we denote the coefficient of the linear terms in $\lambda$ of $N_{r}(\lambda)$ and $D_{r}(\lambda)$ by $N_{r}{ }^{1}$ and $D_{r}{ }^{1}$, respectively, we have:

$$
N_{r}^{1}=(-1)^{r+1} r, \quad D_{r}^{1}=(-1)^{r} \frac{r(r-1)}{2}
$$

Eq. (5) can be written:

$$
\langle F\rangle_{t}=\sum_{i=1}^{r^{t}-1}\left(\frac{\lambda_{i, 2}^{t} \lambda_{i, 3}^{t} \cdots \lambda_{i, r}^{t}+\lambda_{i, 1}^{t} \lambda_{i, 3}^{t} \cdots \lambda_{i, r}^{t}+\cdots+\lambda_{i, 2}^{t} \lambda_{i, 3}^{t} \cdots \lambda_{i, r-1}^{t}}{\lambda_{i, 1}^{t} \lambda_{i, 2}^{t} \cdots \lambda_{i, r}^{t}}\right)+\langle F\rangle_{0}
$$

Since, from Eq. (4), $\lambda_{i, k}^{t}, 1 \leq k \leq r$ are $r$ roots associated with $\lambda_{i}^{t-1}$, we may define a polynomial $Q_{r}(\lambda)$ as follows:

$$
Q_{r}(\lambda):=N_{r}(\lambda)-\lambda_{i}^{t-1} D_{r}(\lambda)
$$

As $Q_{r}(\lambda)=\left(\lambda-\lambda_{i, 1}^{t}\right)\left(\lambda-\lambda_{i, 2}^{t}\right) \ldots\left(\lambda-\lambda_{i, r}^{t}\right)$, from the Vieta's formulas for $Q_{r}(\lambda)=0$, we have

$$
Q_{r}(0)=Q_{r}^{0}=(-1)^{r} \lambda_{i, 1}^{t} \cdots \lambda_{i, r}^{t}
$$


and the linear coefficient on $\lambda$ of $Q_{r}(\lambda)$ is

$$
Q_{r}^{1}=(-1)^{r-1}\left(\lambda_{i, 2}^{t} \lambda_{i, 3}^{t} \cdots \lambda_{i, r}^{t}+\lambda_{i, 1}^{t} \lambda_{i, 3}^{t} \cdots \lambda_{i, r}^{t}+\cdots+\lambda_{i, 2}^{t} \lambda_{i, 3}^{t} \cdots \lambda_{i, r-1}^{t}\right)
$$

On the other hand, from Eq. (9) and using equations (6) and (7) we can obtain the expressions $Q_{r}^{0}$ and $Q_{r}^{1}$ as a function of $r$ and $\lambda_{i}^{t-1}$,

$$
Q_{r}^{0}=N_{r}(0)-\lambda_{i}^{t-1} D_{r}(0)=-\lambda_{i}^{t-1}(-1)^{r-1}=(-1)^{r} \lambda_{i}^{t-1}
$$

and

$$
Q_{r}{ }^{1}=N_{r}{ }^{1}-\lambda_{i}^{t-1} D_{r}{ }^{1}=(-1)^{r+1} r-\lambda_{i}^{t-1}(-1)^{r} \frac{r(r-1)}{2}=(-1)^{r+1}\left(r+\frac{\lambda_{i}^{t-1}}{2} r(r-1)\right)
$$

and finally we have,

$$
\lambda_{i_{1}}^{t} \cdots \lambda_{i_{r}}^{t}=\lambda_{i}^{t-1}
$$

and

$$
\lambda_{i_{2}}^{t} \lambda_{i_{3}}^{t} \cdots \lambda_{i_{r}}^{t}+\lambda_{i_{1}}^{t} \lambda_{i_{3}}^{t} \cdots \lambda_{i_{r}}^{t}+\cdots+\lambda_{i_{2}}^{t} \lambda_{i_{3}}^{t} \cdots \lambda_{i_{r-1}}^{t}=r+\frac{\lambda_{i}^{t-1}}{2} r(r-1)
$$

Substituting these results into Eq. (8)

$$
\begin{aligned}
\langle F\rangle_{t} & =\sum_{i=1}^{r^{t}-1}\left(\frac{r+\frac{\lambda_{i}^{t-1}}{2} r(r-1)}{\lambda_{i}^{t-1}}\right)+\langle F\rangle_{0}=r \sum_{i=1}^{r^{t}-1} \frac{1}{\lambda_{i}^{t-1}}+\sum_{i=1}^{r^{t}-1} \frac{r(r-1)}{2}+\langle F\rangle_{0} \\
& =r\langle F\rangle_{t-1}+\left(r^{t}-1\right) \frac{r(r-1)}{2}+\langle F\rangle_{0}
\end{aligned}
$$

Recall that $\langle F\rangle_{0}=\sum_{i=2}^{r} \frac{1}{\lambda_{i}^{0}}$, where $\lambda_{i}^{0} i \in\{1,2, \ldots, r\}$ are the eigenvalues of $T_{r, 0}=P_{r}$ distinct from 0 . The spectra of $P_{r}$ is well known $[18,31]$ and can be written as $s p\left(P_{r}\right)=$ $\left\{0,4 \sin ^{2}\left(\frac{\pi}{2 r}\right), 4 \sin ^{2}\left(\frac{2 \pi}{2 r}\right), \cdots, 4 \sin ^{2}\left(\frac{(r-1) \pi}{2 r}\right)\right\}$. Therefore, using results from [32], we have:

$$
\langle F\rangle_{0}=\sum_{i=1}^{r-1} \sin ^{-2}\left(\frac{i \pi}{2 r}\right)=\frac{r^{2}-1}{6}
$$

and Eq. (8) gives

$$
\langle F\rangle_{t}=r\langle F\rangle_{t-1}+\left(r^{t}-1\right) \frac{r(r-1)}{2}+\frac{r^{2}-1}{6}
$$

Finally, solving this recurrence equation we obtain the mean first passage time for the generalized deterministic recursive tree $T_{r, t}$ as:

$$
\langle F\rangle_{t}=\frac{1}{6}\left((2 r-1)+((r-2)+3(r-1) t) r^{t+1}\right)
$$


Equation (11) shows the explicit dependence of the MFPT on $t$ and $r$. For $t$ large, $\langle F\rangle_{t} \approx \frac{1}{2}(r-1) t r^{t+1}$ and as, at step $t,|V(G)|=n_{t}=r^{t+1}$, we find for a large GDRT network $(n \rightarrow \infty)$ the following expression:

$$
\langle F\rangle \sim n \log n
$$

This asymptotic dependence of MFPT with the network order can be compared with the MFPT scalings found for other trees, like the T-fractal tree, with $\langle F\rangle \sim n^{2 \ln 6 / \ln 9}[15]$ and the iterative fractal scale-free network, with $\langle F\rangle \sim n^{3 / 2}$ [13]. For scale-free graphs, the existence of hubs improves the MFPT as it is the case of Apollonian networks, where $\langle F\rangle \sim n^{2-\ln 5 / \ln 3}[11]$ or the pseudofractal scale-free web with $\langle F\rangle \sim n^{\ln 2 / \ln 3}[10]$. However,

fractal graphs like the Sierpinski gasket, with $\langle F\rangle \sim n^{\ln 5 / \ln 3}[6]$ or the Koch networks with $\langle F\rangle \sim n[12]$ have a larger MFPT. Finally, Montroll [34] shows that for regular lattices with a large order $n$, and dimensions $d=1, d=2$, and $d=3$, the MFPT is $\langle F\rangle \sim n^{2}$, $\langle F\rangle \sim n \log n$ and $\langle F\rangle \sim n$, respectively.

\section{CONCLUSIONS}

In this paper, we have introduced a method to find the exact analytical expression for the mean first passage time of self-similar recursive trees. The method is based on the relationship between the MFPT and and the Laplacian eigenvalues of the network, but avoids their explicit calculation, resulting in a simple and elegant technique. We have applied the method to find the MFPT for the generalized deterministic recursive trees but it can be adapted to other self-similar tree families. We expect that it will be useful in the study of the MFPT of other tree families, including some fractal trees.

\section{Acknowledgements}

This research has been supported by the Ministry of Science and Innovation, Spain, and the European Regional Development Fund (ERDF) under project MTM2008-06620- C03-01 and by the Catalan Research Council (grant 2009SGR1387).

We thank Zhongzhi Zhang (University of Fudan, China) for his useful comments and 
suggestions which lead to this study.

[1] M.E.J. Newman, SIAM Review 45, 167 (2003).

[2] E. Ravasz and A-L. Barabási, Phys. Rev. E 67, 026112 (2003).

[3] A-L Barabási and Z.N. Oltvai, Nature Rev. Genetics 5101 (2004).

[4] E. Agliari, R. Burioni, D. Cassi, and F. M. Neri, Theor. Chem. Acc. 118, 855 (2007) .

[5] G. Oshanin, O. Bénichou, and A. Blumen, J. Stat. Phys. 112, 541 (2003).

[6] E. M. Bollt and D. ben-Avraham, New J. Phys. 7, 26 (2005).

[7] S. Condamin, O. Bénichou, V. Tejedor, R. Voituriez, and J. Klafter, Nature 450, 77 (2007).

[8] J. J. Kozak and V. Balakrishnan, Phys. Rev. E 65, 021105 (2002).

[9] J. J. Kozak and V. Balakrishnan, Int. J. Bifurcat. Chaos Appl. Sci. Eng. 12, 2379 (2002).

[10] Z. Zhang,Y. Qi, S. Zhou, W. Xie, and J. Guan, Phys. Rev. E 79, 021127 (2009).

[11] Z. Zhang, J. Guan, W. Xie, Y. Qi, and S. Zhou. EPL 86, 10006 (2009).

[12] Z. Zhang, S. Zhou, W. Xie, L. Chen, Y. Lin, and J. Guan. Phys. Rev. E 79, 061113 (2009).

[13] Z. Zhang, W. Xie, S. Zhou, S. Gao, and J. Guan. EPL 88, 10001 (2009).

[14] E. Agliari, Phys. Rev. E 77011128 (2008) .

[15] Z. Zhang, Y. Lin, S. Zhou, B. Wu, and J. Guan, New J. Phys. 11103043 (2009).

[16] D.M Cvetkovic, M. Doob and H. Sachs, Spectra of graphs. Theory and application, New York: Academic Press. 1980.

[17] F.R.K. Chung, Spectral Graph Theory, University of Pennsylvania, Philadelphia, PA, 1997.

[18] B. Mohar, The Laplacian spectrum of graphs, In Graph Theory, Combinatorics, and Applications; Alavi, Y., Chartrand, G., Ollermann, O. R., Schwenk, A. J., Eds.; Wiley: New York, 1991; pp 871-898.

[19] M. Barahona and L. M. Pecora. Phys. Rev. Lett. 89, 054101 (2002).

[20] F. Comellas and S. Gago. J. Phys. A: Math. Theor. 40, 4483 (2007).

[21] A. Arenas, A. Diaz-Guilera, J. Kurths, Y. Moreno, and C. Zhou, Physics Reports 469 (2008) 93-153.

[22] L. Barrière, F. Comellas, C. Dalfó, and M. A. Fiol, Linear Algebra Appl. 428, 1499 (2008).

[23] L. Barrière, F. Comellas, C. Dalfó, and M.A. Fiol, Discrete Appl. Math. 157, 36 (2009).

[24] L. Barrière, F. Comellas, C. Dalfó, and M.A. Fiol, Linear and Multilinear Algebra. 57, 695 
(2009).

[25] Y. Qi, Z. Zhang, B. Ding, S. Zhou, and J. Guan, J. Phys. A: Math. Theor. 42, 165103 (2009).

[26] Z. Zhang,Y. Qi, S. Zhou,Y. Lin, and J. Guan, Phys. Rev. E 80, 016104 (2009).

[27] S. Jung, S. Kim, and B. Kahng, Phys. Rev. E 65056101 (2002).

[28] A. K. Chandra, P. Raghavan, W. L. Ruzzo, R. Smolensky, The electrical resistance of a graph captures its commute and cover times. STOC '89: Proceedings of the twenty-first annual ACM symposium on theory of computing, pp. 574 - 586 (1989)

[29] D.J. Klein and M. Randić, J. Math. Chem. 12, 81 (1993).

[30] I. Gutman, B. Mohar. J. Chem. Inf. Comput. Sci. 36, 982-985 (1996).

[31] W. N. Anderson Jr. and T. Morley, Linear and Multilinear Algebra. 18, 141 (1985).

[32] F. Calogero, and A.M.Perelomov, Linear Algebra Appl. 25, 91 (1979) .

[33] Z.-G. Huang, X.-J. Xu, Z.-X. Wu, and Y.-H. Wang, Eur. Phys. J. B 51, 549 (2006).

[34] E. W. Montroll, J. Math. Phys. 10, 753 (1969). 